\title{
Análise Factorial Confirmatória da Escala de Sofrimento para Cuidadores em Enfermeiros Portugueses nos Cuidados de Saúde Primários
}

\author{
Caregiver Grief Scale Confirmatory Factor Analysis in Portuguese Primary Health \\ Care Nurses
}

José Vasconcelos-Raposo ${ }^{1}$, Luísa Pires ${ }^{2}$, Maria João Monteiro ${ }^{3}$ e Carla Maria Teixeira ${ }^{4}$

\begin{abstract}
Resumo
O presente estudo tem como objectivo adaptar e validar a Escala de Sofrimento para Cuidadores (Caregiver Grief Scale), previamente validada para a população portuguesa nos cuidadores formais e informais por Vasconcelos-Raposo, Ribeiro, Silva, Santos e Teixeira (2019). A amostra foi constituída por 100 enfermeiros nos Cuidados de Saúde Primários (CSP). Para a análise das propriedades psicométricas do instrumento, recorreu-se à Análise Factorial Confirmatória. Os resultados evidenciaram uma estrutura de três factores (Dor Emocional, Perda Relacional e Evitamento), o que contraria a versão original em alemão, assim como a validação prévia em português. A versão para enfermeiros apresentou os seguintes índices de ajustamento: $\chi^{2} / \mathrm{df}=1.609 ; \mathrm{CFI}=.940 ; \mathrm{GFI}=.901 ; \mathrm{RMSEA}=.078 ; \mathrm{AIC}=115.965$, revelando boa fiabilidade compósita (.923) e validade discriminante. Em conclusão, a Escala de Sofrimento para Cuidadores (CGS) apresenta robustez para ser utilizada no estudo do sofrimento dos enfermeiros portugueses nos CSP.
\end{abstract}

Palavras-chave: sofrimento psíquico, análise factorial confirmatória, cuidados de saúde primários, estudos de validação

\begin{abstract}
The present study aims to adapt and validate the Caregiver Grief Scale (CGS), previously validated for the Portuguese population for formal and informal caregivers by Vasconcelos-Raposo, Ribeiro, Silva, Santos, and Teixeira (2019). The sample consisted of 100 nurses in the Primary Health Care Services (CSP). For the analysis of the psychometric properties of the instrument, Confirmatory Factor Analysis was used. The results showed a structure of three factors (Emotional Pain, Relational Loss, and Avoidance), which contradicts the original German version, as well as the earlier validation in Portuguese. The nurses' version presented the following adjustment indices: $\chi^{2} / \mathrm{df}=1.609 ; \mathrm{CFI}=.940 ; \mathrm{GFI}=.901 ; \mathrm{RMSEA}=.078$; AIC $=115,965$, showing good composite reliability (.923) and discriminant validity. In conclusion, the Caregiver Grief Scale presents robustness to be used in the study of the grief of Portuguese nurses in Primary Health Care.
\end{abstract}

Keywords: psychological suffering, confirmatory factor analysis, primary health care, validation study

\footnotetext{
${ }^{1}$ Doutorado em Psicologia do Desporto, Exercício e Saúde, Professor Catedrático, ECHS Universidade de Trás-os-Montes e Alto Douro, INESCTEC MASSIVE Lab., Quinta de Prados 5000-801 Vila Real, Portugal. Tel.: (+351) 259350000. E-mail: jvraposo@ utad.pt

${ }^{2}$ Mestre em Enfermagem Comunitária. Centro Hospital de Trás-os-Montes, Enfermeira, R. dos Lagoeiros 43, Vila Real, Portugal. Tel.: (+351) 259300500. E-mail: lpires.luisamaria@gmail.com

${ }^{3}$ Doutorada em Saúde. Escola Superior de Saúde, Universidade de Trás-os-Montes e Alto Douro, Quinta de Prados 5000-801 Vila Real, Portugal. Tel.: (+351) 259350 967. E-mail: marijosilva@ @apo.pt

${ }^{4}$ Doutorada em Psicologia. Prof. Auxiliar, ECHS Universidade de Trás-os-Montes e Alto Douro, MASSIVE Lab., Quinta de Prados 5000801, Vila Real. (+351) 259350000. E-mail: cteixeir@utad.pt

Revista Iberoamericana de Diagnóstico y Evaluación - e Avaliação Psicológica. RIDEP · No57 · Vol.4 · 177-190 • 2020

ISSN: 1135-3848 print /2183-6051online
} 


\section{Introdução}

A problemática do sofrimento tem vindo a ser estudada desde a cultura ancestral dos nossos antepassados, embora com importância e significados diferentes. De acordo com Cerqueira (2011), apenas no século XVI foi atribuído significado à experiência de sofrimento, tal como a uma experiência de dor. Hoje, o sofrimento é reconhecido como algo que afecta a unidade e integridade da pessoa, provocando uma ruptura na sua identidade e, consequentemente, no bem-estar e na saúde.

Acompanhar o percurso do doente e tomar consciência do sofrimento do outro, de quem cuidamos, face ao resultado da intervenção profissional, poderá resultar tanto em satisfação como em sofrimento. Apresenta-se como fonte de satisfação quando traz realização pessoal e o profissional desenvolve as suas potencialidades humanas através do exercício da profissão, sentindo-se útil à sociedade (Paula et al., 2010). Um prestador de cuidados em sofrimento é alguém diminuído nas suas capacidades. São vários os estudos que associam sintomas físicos e emocionais tais como, cefaleias, fadiga, tristeza, raiva, culpa, ansiedade, depressão, fenómenos de stresse e burnout, aos profissionais de saúde (Adwan, 2014; Chan, Livingston, Jones, \& Sampson, 2012), o que vem reforçar a necessidade de estudos sobre o sofrimento em prestadores de cuidados de saúde. O cuidar é uma atitude que envolve responsabilidade, preocupação e afecto com o outro, sendo por isso, algo que vai muito para além do ato técnico da profissão (Coelho \& Ferreira, 2015; Gama, 2013; Hedler, Santos, Faleiros, \& Almeida, 2016; Machado \& Merlo, 2008).

Sendo um fenómeno biopsicossocial e espiritual, o sofrimento está presente em todos os contextos associados à doença. Não tem só repercussões para o doente/família, mas também para os profissionais de saúde (Peixoto \& Borges, 2011; Wenzel, Shaha, Klimmek, \& Krumm, 2011; Wisekal, 2015).

Lindberg (2012) refere que na presença de um doente em sofrimento ou aquando da sua morte, os sentimentos de perda são inevitáveis, sendo que esses sentimentos poderão ser maiores ou menores, dependendo do tempo passado com o doente e do tipo de relação estabelecida com a família deste. No entanto, a autora sublinha que cada morte deixará sempre uma marca nos enfermeiros que lhe prestaram cuidados.

O sofrimento é algo difícil de expressar por palavras, é algo intocável e invisível, que não se transmite, apenas existe singularmente no sujeito (Viana, 2014). Travelbee (2004) refere um sentimento de desprazer que vai do simples desconforto mental, físico ou espiritual transitórios até à extrema angústia, às fases para além da angústia, à fase maligna de 'não cuidado' desesperante e à fase terminal de indiferença apática. Krikorian, Limonero, Román, Vargas e Palacio (2014), definem o sofrimento como uma experiência multidimensional e dinâmica de stresse severo que ocorre perante eventos de ameaça à integridade da pessoa no seu todo, podendo conduzir à exaustão.

O sofrimento advém da experiência pessoal, é individual, subjectivo e está associado a situações que ameaçam a integridade da pessoa, podendo estar relacionado com um sintoma ou um processo (físico ou de outra natureza), acompanhado por uma série de emoções intensas das quais se destacam a tristeza, a angústia, o medo, o abandono e o desespero, sendo sentido por cada pessoa de forma diferente e em vários momentos da vida, ainda que apresentem a mesma sintomatologia (Encarnação, Oliveira, \& Martins, 2015).

O enfermeiro é um profissional, no entanto, não podemos deixar de referir que é também uma pessoa, caracterizada pela sua individualidade e singularidade comportamental, emocional e cognitiva (Coelho \& Ferreira, 2015). Numa actividade profissional em que a relação interpessoal é uma componente imprescindível para a qualidade dos cuidados e que envolve sentimentos e emoções, o contacto com o sofrimento do doente/família é, para o enfermeiro, fonte de sofrimento (Borges, 2005). O sentimento de não querer envolver-se para não sofrer proporciona, inevitavelmente, sofrimento pelo conflito gerado entre o agir com a razão ou com a emoção, ou o sentimento de compaixão para com o outro (Rodrigues \& Silva, 2016).

De acordo com estas conceptualizações do sofrimento, seria espectável que esta componente inerente à actividade profissional do enfermeiro 
estivesse amplamente estudada. No entanto, isto não se verifica. Este facto é, ainda, agravado por serem escassos os instrumentos devidamente validados para avaliar o sofrimento em cuidadores formais ou informais, quer na literatura nacional quer na internacional.

Da revisão de literatura percebe-se que face à exposição e ao acompanhamento do sofrimento de quem se cuida leva a graus mais acentuados de sofrimento naquele que cuida/cuidou de uma pessoa com doença ou problema de saúde (Pires, Monteiro, \& Vasconcelos-Raposo, 2020). Não basta afirmar que os cuidadores são uns heróis invisíveis e solitários, pois nem sempre são vistos como fundamentais ou valorizados como cuidadores, quer seja pela família, quer pelos serviços de saúde (Luzardo \& Waldman, 2004). Parece existir consenso quando se afirma a necessidade de avaliar o sofrimento dos cuidadores no sentido de consciencializar estes e as entidades empregadoras mas, para isso, urge encontrar instrumentos que o permitam fazer de forma fiável e válida.

Ao longo dos últimos anos temos assistido, na literatura internacional, a um crescente volume de publicações que abordam a temática do sofrimento em prestadores de cuidados de saúde, nomeadamente nos enfermeiros e múltiplos contextos de intervenção profissional ( ver Allie et al., 2018; Almeida et al, 2016; Oliveira et al., 2018; Vega et al., 2017; Lin, Liu, \& Chiang, 2020; Mork \& Moules, 2019; Silva \& Neto, 2018) assim como o impacto da fadiga de compaixão (compation fadigue) na saúde dos enfermeiros (Adimando, 2018). A literatura em língua portuguesa apresenta-se mais rica em estudos realizados no Brasil (i.e. Caldeira \& Santos, 2019; Campos, David, \& Souza, 2014; Paula et al., 2010; Pimenta et al., 2020).

Em Portugal (Pires et al., 2020; Schaefer, 2017) a maioria das investigações sobre o sofrimento tem-se focado no estudo do sofrimento de doentes ou cuidadores informais, descurando os profissionais de saúde, nomeadamente os enfermeiros que diariamente se deparam com o sofrimento e até perda de vida daqueles de quem cuidam, pelo que com este trabalho procuramos colmatar, em parte, esta lacuna.

O presente estudo centra-se nos prestadores de cuidados de saúde primários, uma vez que são estes que "representam o primeiro nível de contacto com o sistema nacional de saúde para os indivíduos, as famílias e a comunidade, trazendo os cuidados de saúde tão próximo quanto possível para os locais onde as pessoas vivem $e$ trabalham" (Conselho Internacional de Enfermeiros, 2008, p. 1). A este nível de envolvimento o enfermeiro desenvolve uma relação de proximidade mais intensa com as famílias e com a comunidade em geral. Graças a estas dinâmicas relacionais o enfermeiro, tende a estar mais alerta para as condições financeiras, alojamento, educação e meios de suporte social que, de alguma forma, condicionam o estado de saúde do indivíduo e as consequências que daí podem advir para a família e para a comunidade. Por essa razão, importa estudar qual o grau de sofrimento a que estes profissionais estão sujeitos.

Tendo em consideração a presença da Caregiver Grief Scale (CGS) na literatura da especialidade, já traduzida e validada para cuidadores formais e informais na população portuguesa, por Vasconcelos-Raposo, Ribeiro, Silva, Santos e Teixeira (2019), com um modelo unifactorial e revelando bom índice de consistência interna, pretendemos adaptar e validar esta escala para os enfermeiros portugueses, dada a ausência de instrumentos validados para esta especialidade de cuidadores na língua portuguesa (Europa). É uma escala breve e de fácil utilização. A sua escolha teve, ainda, como critério o facto de esta apresentar itens que questionam directamente sobre a dor e a perda, permitindo avaliar o sofrimento e integrar diferentes aspectos do sofrimento nos prestadores de cuidados, tais como os sentimentos de perda, a reacção emocional a essa perda, a tendência a evitar ou a aceitá-la e o sofrimento vivido.

A CGS foi, inicialmente, construída e validada numa amostra alemã, constituída por cuidadores de pessoas com demência. Na versão original da CGS, foi possível fazer a distinção entre subgrupos de cuidadores. Por exemplo, prevê que cônjuges cuidadores apresentem maior intensidade de luto e que cuidadores que vivem com a pessoa cuidada apresentem valores mais elevados na CGS, uma vez que, com regularidade, presenciam perdas na vida quotidiana, aumentando o sofrimento experienciado. É a sensibilidade a este tipo de factores e às diferenças 
existentes entre os subgrupos de cuidadores que torna a CGS um instrumento vantajoso (Meichsner, Schinköthe, \& Wilz, 2016).

Até ao surgimento da CGS, o único instrumento desenvolvido para avaliar a dor do cuidador era o Marwit - Meuser Caregiver Grief Inventory. Este inventário inclui itens que avaliam a depressão e a sobrecarga, contudo, não abrange directamente a evasão do sofrimento dos cuidadores (Meichsner et al., 2016). Para a construção da versão original da CGS, na Alemanha, foi utilizada uma amostra de 229 cuidadores informais e foi validada para os mesmos com quatro factores (Dor Emocional, Perda Relacional, Perda Absoluta e Aceitação da Perda). Na versão alemã, com quatro factores, o factor Dor Emocional diz respeito à experiência de sofrimento e outras emoções desagradáveis, vivenciadas no presente e relacionadas com a perda de alguém. O factor Perda Relacional, refere-se às perdas relativas ao relacionamento e experiências partilhadas com a pessoa cuidada quando esta ainda era saudável. O factor Perda Absoluta, refere-se ao fim do relacionamento com a pessoa que carece de cuidado e antecipação por parte do cuidador de um futuro sem essa pessoa. E, por fim, o factor Aceitação da Perda reflecte a aceitação por parte do cuidador da doença/problema da pessoa cuidada e da reacção ao luto. Esta aceitação inclui a expressão livre de sofrimento, porque os cuidadores, muitas vezes, evitam expressar ou mesmo sentir tristeza durante o período em que a pessoa que carece de cuidado está viva (Meichsner et al., 2016). A validação deste instrumento está em curso para a população inglesa, com o intuito de tornar a escala disponível para uso internacional (Meichsner et al., 2016). Para a língua portuguesa, já foi adaptada e validada para cuidadores formais e informais $(n=150)$ por Vasconcelos-Raposo et al. (2019) e resultou numa estrutura unifactorial que os autores designaram de Sofrimento. Na versão portuguesa, a estrutura multifactorial proposta por Meichsner não foi confirmada por falta de validade discriminante. Assim, foi proposto o conceito de sofrimento de forma a englobar os múltiplos aspectos referidos na versão original alemã, sendo argumentado que a não diferenciação das diferentes dimensões do sofrimento se devem à cultura.
Os instrumentos psicológicos, ao longo das décadas, têm-se revelado como um dos instrumentos mais valiosos para a prática clínica (diagnóstico) e a investigação nomotética (Prieto, Muniz, Almeida, \& Bartram, 1999). No entanto, a utilização destes apresenta-se problemática e um dos aspectos mais relevantes é a inadequada adaptação cultural e validação dos instrumentos em causa (Seabra-Santos, Almiro, Simões, \& Almeida, 2019), algo que o presente trabalho visa ultrapassar. Devido à falta de instrumentos que avaliem o sofrimento dos enfermeiros em Portugal, o objectivo geral deste estudo é adaptar e validar a Escala de Sofrimento para Cuidadores (Caregiver Grief Scale) para os enfermeiros portugueses que exercem funções nos Cuidados de Saúde Primários. Como objectivo específico pretende-se determinar as propriedades psicométricas da Escala de Sofrimento para Cuidadores (CGS) numa amostra de enfermeiros, recorrendo à Análise Factorial Confirmatória (AFC).

\section{Método}

O presente estudo é quantitativo e correlacional e visa a adaptação e validação da Escala de Sofrimento para Cuidadores (CGS) para os enfermeiros portugueses nos CSP com recurso à AFC. A amostra foi constituída por 100 enfermeiros que exercem a sua atividade profissional num Agrupamento de Centros de Saúde (ACeS) do interior Norte de Portugal, representando $90,09 \%$ da população em estudo e $100 \%$ no que se refere ao sexo masculino.

No que respeita à caracterização sociodemográfica e profissional da amostra em estudo, com 10 sujeitos do sexo masculino e 90 do sexo feminino, a idade mínima dos participantes foi de 33 anos e a idade máxima de 63 anos. A média de idades foi $43.9 \pm 7.45$ anos (Quadro 1). Constatamos o desequilíbrio da variável "sexo", mas essa é a realidade da profissão aqui em causa. A técnica de amostragem foi não aleatória e de conveniência. Os dados foram recolhidos entre 19 de fevereiro e 5 de março de 2018. Como critérios de inclusão para o estudo foram definidos: enfermeiros de ambos os sexos, de nacionalidade portuguesa e que aceitaram participar no estudo 
Quadro 1. Caracterização sociodemográfica e profissional da amostra de enfermeiros

\begin{tabular}{|c|c|c|c|}
\hline & & $\begin{array}{c}\mathrm{n} \\
(\mathrm{N}=100)\end{array}$ & $\%$ \\
\hline Sexo & $\begin{array}{l}\text { Masculino } \\
\text { Feminino }\end{array}$ & $\begin{array}{l}10 \\
90\end{array}$ & $\begin{array}{l}10.0 \\
90.0\end{array}$ \\
\hline Grupo etário & $\begin{array}{c}30-40 \text { anos } \\
41-51 \text { anos } \\
52-62 \text { anos } \\
>62 \text { anos }\end{array}$ & $\begin{array}{c}38 \\
43 \\
18 \\
1\end{array}$ & $\begin{array}{c}38.0 \\
43.0 \\
18.0 \\
1.0\end{array}$ \\
\hline Habilitações literárias & $\begin{array}{l}\text { Licenciatura } \\
\text { Mestrado }\end{array}$ & $\begin{array}{l}80 \\
20\end{array}$ & $\begin{array}{l}80.0 \\
20.0\end{array}$ \\
\hline Unidade funcional de prestação de cuidados & $\begin{array}{c}\text { UCC } \\
\text { USF } \\
\text { UCSP }\end{array}$ & $\begin{array}{l}37 \\
35 \\
28\end{array}$ & $\begin{array}{l}37.0 \\
35.0 \\
28.0\end{array}$ \\
\hline Cuidador informal $(\mathrm{n}=36)$ & $\begin{array}{l}\text { Sim } \\
\text { Não }\end{array}$ & $\begin{array}{l}36 \\
64\end{array}$ & $\begin{array}{l}36.0 \\
64.0\end{array}$ \\
\hline Tempo de cuidador informal $(n=36)$ & $\begin{array}{c}<1 \text { ano } \\
1-3 \text { anos } \\
4-6 \text { anos } \\
7-9 \text { anos } \\
>10 \text { anos }\end{array}$ & $\begin{array}{c}7 \\
17 \\
5 \\
4 \\
3\end{array}$ & $\begin{array}{l}19.45 \\
47.22 \\
13.89 \\
11.11 \\
8.33\end{array}$ \\
\hline Número de pessoas cuidadas $(\mathrm{n}=36)$ & $\begin{array}{l}1 \text { pessoa } \\
2 \text { pessoas } \\
3 \text { pessoas } \\
4 \text { pessoas }\end{array}$ & $\begin{array}{c}24 \\
7 \\
3 \\
2\end{array}$ & $\begin{array}{c}66.67 \\
19.44 \\
8.33 \\
5.56\end{array}$ \\
\hline De quem cuidou $(n=36)$ & $\begin{array}{c}\text { Companheiro/a } \\
\text { Pais } \\
\text { Filhos } \\
\text { Sogros } \\
\text { Avós }\end{array}$ & $\begin{array}{c}1 \\
22 \\
0 \\
4 \\
9\end{array}$ & $\begin{array}{c}2.78 \\
61.11 \\
0.0 \\
11.11 \\
25.0\end{array}$ \\
\hline Vive/viveu com a pessoa cuidada $(\mathrm{n}=36)$ & $\begin{array}{l}\text { Sim } \\
\text { Não }\end{array}$ & $\begin{array}{l}21 \\
15\end{array}$ & $\begin{array}{l}58.33 \\
41.67\end{array}$ \\
\hline
\end{tabular}

UCC: Unidade de Cuidados na Comunidade; USF: Unidade de Saúde Familiar; UCSP: Unidade de Cuidados de Saúde Personalizados.

de forma voluntária. E como critérios de exclusão: não ter respondido a pelo menos $80 \%$ do instrumento de recolha de dados e não estar directamente implicado na prestação de cuidados de enfermagem ao doente.

\section{Instrumento}

Para o presente estudo foi elaborado um questionário que teve por objectivo a caracterização sociodemográfica e profissional da amostra, seguindo-se as questões da CGS para avaliar o sofrimento dos enfermeiros.

A CGS foi desenvolvida e validada na Alemanha por Meichsner et al. (2016). No processo de validação foram testadas duas estruturas: uma com três factores, que foi rejeitada e outra com quatro factores que foi validada. $\mathrm{Na}$ sua versão original, esta escala é constituída por 11 itens que avaliam as seguintes quatro dimensões do sofrimento do cuidador: Dor Emocional, Perda
Relacional, Perda Absoluta e Aceitação da Perda.

$\mathrm{Na}$ versão portuguesa, o modelo alemão de quatro factores foi rejeitado. Posteriormente, foi testado um modelo unifactorial que, após correcções, foi validado para cuidadores formais e informais (Vasconcelos-Raposo et al., 2019). O factor foi designado de Sofrimento e manteve os 11 itens da escala alemã. As respostas estão organizadas numa escala do tipo Likert: 1. Discordo totalmente, 2. Discordo, 3. Concordo moderadamente, 4. Concordo e 5. Concordo totalmente.

\section{Procedimento}

Em primeiro lugar, de acordo com as recomendações, procedemos à operacionalização e adaptação semântica para enfermeiros da versão portuguesa da CGS, já validada para cuidadores formais e informais. A versão original desta escala foi traduzida e adaptada para a língua portuguesa aquando do trabalho realizado por Vasconcelos- 
Raposo et al. (2019), sobre “Validação da versão portuguesa da Caregiver Grief Scale/ Escala de Sofrimento para Cuidadores". Para os propósitos do presente estudo, procedemos à adaptação semântica dos itens à realidade do contexto profissional a ser estudado, com o intuito de conseguir uma equivalência a nível conceitual, de mensuração e a nível funcional, seguindo as recomendações de Reichenhim e Moraes (2007). Para este efeito, foram consultados especialistas na área da enfermagem e da psicometria. Houve sempre o maior cuidado ao reformular cada um dos itens de forma a se adequarem às características do relacionamento enfermeiro doente e que a linguagem fosse acessível e de fácil compreensão para todos. Posteriormente, o questionário foi aplicado a vinte enfermeiros que exerciam funções em contextos similares ao da população alvo do estudo. A partir das evidências encontradas nesta aplicação, procedeu-se ao ajustamento recomendado acrescentando nos itens a expressão "doentes ao meu cuidado".

$\mathrm{O}$ questionário foi aplicado aos enfermeiros que exercem a sua actividade profissional em CSP num ACeS do interior Norte de Portugal após parecer favorável da Comissão de Ética para a Saúde (CES) da Administração Regional de Saúde dessa região, bem como a autorização dos autores da versão portuguesa da escala a aplicar na investigação. Os questionários foram entregues num envelope aberto ao enfermeiro chefe ou responsável de cada unidade funcional que, por sua vez, os distribuiu pelos enfermeiros das referidas unidades. Os questionários seguiam acompanhados de uma declaração onde eram referidos os objectivos do estudo, garantida a confidencialidade e anonimato, salientando-se que os dados se destinavam apenas a fins de investigação e respeitando os princípios éticos da mesma. Os participantes no estudo foram, também, informados que poderiam desistir a qualquer momento.

Foram distribuídos 111 questionários e recolhidos 100 , o que corresponde a $90.09 \%$ da população. Os restantes onze não foram devolvidos pelos participantes no prazo estabelecido. Os questionários foram preenchidos, colocados no envelope fechado e entregues ao responsável pela unidade funcional. Nenhum dos participantes relatou qualquer incidente que pudesse ter influenciado o preenchimento do questionário. Relativamente à validade externa, tivemos como referência a participação activa dos autores da validação prévia em português. Após a recolha de dados, foi verificado se todos os itens tinham sido respondidos de acordo com as instruções. Todos os dados recolhidos através dos questionários foram aceites para a construção da base de dados final. Os dados recolhidos foram sujeitos a todas as análises estatísticas consideradas pertinentes.

\section{Análise estatística}

Para os propósitos em causa, recorremos à Análise Factorial Confirmatória (AFC) e, consequentemente, foram calculados os seguintes indicadores: validade factorial, validade convergente, validade discriminante e consistência interna, na amostra em estudo de enfermeiros dos CSP.

O tratamento dos dados obtidos foi efectuado através do software SPSS (Statistical Package for the Social Sciences), versão 24 e do AMOS (Analysis of Moments Structures), versão 25. Inicialmente, foi realizada a análise de estatísticas descritivas referentes aos itens das dimensões, bem como às variáveis em análise, a partir da Média e do Desvio Padrão. A análise da simetria da distribuição de frequências (normalidade univariada) foi realizada através dos coeficientes Skewness (assimetria) e Kurtosis (achatamento), sendo que os valores devem estar compreendidos entre -1 e 1, para que seja verificada a normalidade dos dados (Marôco, 2014). Foi também calculado o grau de consistência interna das dimensões com recurso ao Alfa de Cronbach para avaliar a fiabilidade do constructo. Ainda segundo o mesmo autor, valores de Alfa de Cronbach superiores a .90 indicam uma consistência interna muito boa, entre .80 e .90 boa, entre .70 e .80 satisfatória e inferiores a .60 baixa.

Começámos por avaliar as estruturas factoriais propostas nos estudos com a amostra alemã e com a amostra portuguesa não se tendo obtido indicadores satisfatórios. Seguiu-se uma Análise Factorial Exploratória (AFE) com a amostra do nosso estudo. A estrutura que resultou da AFE foi, consequentemente, submetida a uma AFC para, posteriormente, compararmos todas as 
Quadro 2. Indicadores das estruturas factoriais previamente propostas na literatura

\begin{tabular}{|c|c|c|c|c|c|}
\hline & $\chi^{2 / d f}$ & CFI & GFI & RMSEA & AIC \\
\hline $\begin{array}{l}\text { Modelo original com } 3 \text { fatores testado (não validado) na Alemanha } \\
\text { por Meichsner et al. (2016) }\end{array}$ & 2.231 & .913 & & .104 & 3776.695 \\
\hline $\begin{array}{l}\text { Modelo original com } 4 \text { fatores testado e validado na Alemanha por } \\
\text { Meichsner et al. (2016) }\end{array}$ & 1.394 & .961 & & .072 & 3436.165 \\
\hline $\begin{array}{l}\text { Modelo unifatorial testado (não validado) em Portugal por } \\
\text { Vasconcelos-Raposo et al. (2019) }\end{array}$ & 5.450 & .849 & .784 & .173 & 283.818 \\
\hline $\begin{array}{l}\text { Modelo unifactorial corrigido e validado em Portugal por } \\
\text { Vasconcelos-Raposo et al. (2019) }\end{array}$ & 2.023 & .970 & .923 & .083 & 110.724 \\
\hline
\end{tabular}

estruturas estudadas e decidirmos pela que melhor se adequa à amostra de enfermeiros nos CSP. Relativamente aos índices de modificação, foram atendidas as correlações de itens com valores superiores a 10. Para os modelos testados, foi utilizado o método de estimação por Maximum Likelihood e, no sentido de verificar a adequabilidade do modelo aos dados, foram usadas as seguintes medidas de avaliação do ajustamento: Ratio Chi-square statistics/degrees of fredoom ( $\left.\chi^{2} / \mathrm{df}\right)$, Comparative Fit Índex (CFI), Goodness of Fit Índex (GFI), Root Mean Square Error of Approximation (RMSEA) e Akaike Information Criterion (AIC). $\mathrm{O} \chi^{2} / \mathrm{df}$ é utilizado como índice de ajustamento do modelo, mas não há consenso sobre o critério para um ajuste adequado, sugerindo-se valores abaixo de 5. O CFI avalia a adequação do modelo em relação ao modelo independente. O GFI avalia as proporções de variância e co-variância simultaneamente explicadas pelo modelo. $\mathrm{O}$ RMSEA averigua a discrepância entre as matrizes estimadas e as observadas, valores superiores a .10 indicam uma adequação inaceitável e, consequentemente, recomenda-se a rejeição do modelo. O AIC é utilizado para comparação entre modelos, valores menores sugerem melhor ajuste do modelo quando comparados aos demais (Marôco, 2014). No presente estudo este indicador assume um carácter secundário servindo para optar entre modelos que se apresentem igualmente válidos.

\section{Resultados}

As análises de todas as estruturas factoriais previamente descritas na literatura relativas ao processo de validação da CGS (Quadro 2) foram testadas, tendo-se obtido os valores descritos no Quadro 3.
Face à divergência dos modelos já validados, com base na amostra do nosso estudo, começamos por submeter à prova a estrutura multifactorial proposta na versão alemã de quatro factores, tendo-se obtido os seguintes valores de $\chi^{2} / \mathrm{df}=2.186 ; \mathrm{CFI}=.891 ; \mathrm{GFI}=.872 ; \mathrm{RMSEA}=.109$ e AIC=139.074 (Quadro 3). Este modelo foi rejeitado porque de acordo com os critérios de aceitação dos modelos, sempre que o valor de RMSEA seja superior a .10 rejeita-se o modelo, independentemente dos valores obtidos nos outros indicadores (Marôco, 2014).

Seguiu-se o teste ao modelo unifactorial, já validado para uma amostra de 150 cuidadores formais e informais, na população portuguesa por Vasconcelos-Raposo et al. (2019). Este modelo quando testado com a amostra de 100 enfermeiros revelou uma qualidade de ajustamento sofrível para o $\chi^{2} / \mathrm{df}$ (3.439), ajustamento mau para o CFI (.741) e GFI (.769) e inaceitável para o RMSEA (0.157) (Quadro 3), pelo que, também, foi rejeitado por razões idênticas ao que se verificou no modelo de quatro factores.

Após testar o modelo unifactorial proposto por Vasconcelos-Raposo et al. (2019), procedemos a alguns ajustamentos, nomeadamente ao nível dos erros (e1-e2; e1-e3; e2-e9; e9-e10; e9-e11 e e10e11), todos apresentaram valores superiores a 10 . Foram feitas as correcções entre os erros referidos e, após estas, o modelo unifactorial ajustado à amostra em estudo revelou uma qualidade de ajustamento bom para o $\chi^{2} / \mathrm{df}(1.781)$ e para o CFI (.929), um ajustamento sofrível para o GFI (.895) e um ajustamento aceitável para o RMSEA (.089) (Quadro 3).

Face à inadequação dos modelos testados, procedeu-se à realização de uma AFE, que evidenciou a existência de três factores, que explicavam $66.69 \%$ da variância. Esta estrutura 
Quadro 3. Resultados das diferentes propostas estruturais obtidas com a amostra de enfermeiros nos CSP

\begin{tabular}{|c|c|c|c|c|c|}
\hline & $\chi^{2} / \mathrm{df}$ & CFI & GFI & RMSEA & AIC \\
\hline $\begin{array}{l}\text { Modelo original com } 4 \text { fatores validado na Alemanha por Meichsner et al. } \\
\text { (2016) }\end{array}$ & 2.186 & .891 & .872 & .109 & 139.074 \\
\hline Modelo unifatorial validado em Portugal por Vasconcelos Raposo et al. (2019) & 3.439 & .741 & .769 & .157 & 195.327 \\
\hline Modelo unifatorial ajustado & 1.781 & .929 & .895 & .089 & 123.66 \\
\hline Modelo multifatorial com 3 fatores validado para enfermeiros nos CSP & 1.609 & .940 & .901 & .078 & 115.965 \\
\hline
\end{tabular}

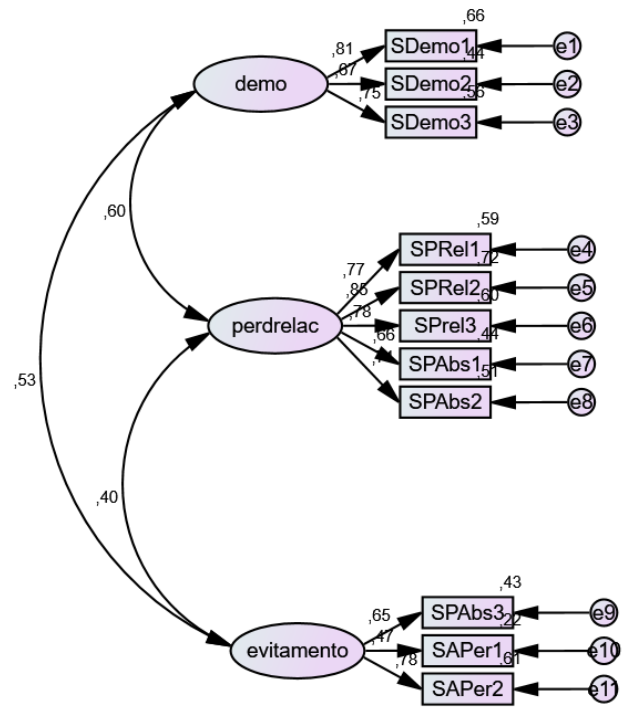

Figura 1. Estrutura fatorial da versão portuguesa da Escala de Sofrimento para Cuidadores (CGS) em enfermeiros nos CSP

mantinha o factor Dor Emocional e o factor Perda Relacional, mas havia itens do factor Perda Absoluta e Aceitação da Perda do modelo original, sugerido na versão alemã de 4 factores, que se juntavam numa subescala a qual se designou por Evitamento.

Foi realizada a análise descritiva inicial no presente estudo, para verificar a distribuição normal das variáveis dependentes, através dos valores de Skewness (assimetria) e Kurtosis (achatamento). Os critérios de normalidade de assimetria variaram entre -.039 e .220 e os de achatamento variam entre -.584 e -.105 , demonstrando que há normalidade na distribuição dos dados, uma vez que todos se encontram no intervalo entre -1 e 1 .

Após a confirmação da normalidade dos dados, esta estrutura foi submetida a uma AFC para, posteriormente, compararmos todas as estruturas estudadas e decidirmos pela que melhor se adequava à amostra de enfermeiros nos CSP.
Para o processo de selecção do modelo que melhor se adequa à amostra de enfermeiros, tivemos em consideração os indicadores de todas as estruturas propostas nas validações previamente encontradas na literatura (Quadro 2).

O modelo multifactorial de três factores (Dor Emocional, Perda Relacional e Evitamento), foi o que apresentou índices de qualidade de ajustamento globais mais adequados: $\square^{2} / \mathrm{df}=1.609$, CFI $=.940$, GFI $=.901$, RMSEA $=.078$ e $\mathrm{AIC}=115.965$, pelo que foi adoptado como estrutura de referência para estudos com enfermeiros prestadores de Cuidados de Saúde Primários (Figura 1).

A validade do constructo é a propriedade do instrumento de medida que avalia se este mede a variável latente que, realmente, se pretende medir e cujos índices de adequação apresentamos (Quadro 3). Esta, por sua vez, é determinada por três subcomponentes: validade factorial, validade convergente e validade discriminante. A validade factorial é dada pelos pesos factoriais estandardizados de todos os itens que, no caso do presente estudo, foram definidos como iguais ou superiores a .5. Pela análise da ilustração do modelo, verificamos que este apresenta validade factorial (Figura 1).

A validade convergente ocorre quando os itens que constituem o constructo apresentam correlações positivas e elevadas entre si. Assim, para que esta seja considerada adequada, o seu valor deve ser igual ou superior a .5. A validade convergente foi avaliada através da média de variâncias dos itens que $\mathrm{o}$ factor explica ou Variância Extraída Média (VEM) pelo factor (Marôco, 2014). Neste modelo multifactorial, o valor obtido de VEM para o factor Dor Emocional foi de .556, para o factor Perda Relacional foi de .573 e para o factor Evitamento foi de .417. No entanto, se fosse eliminado o item 10 (SAPer1), 
que apresenta uma carga factorial de .47 , ligeiramente abaixo do estabelecido como critério inicial (.5), a VEM para o factor Evitamento seria de .515 , logo apresentando validade convergente adequada. Foi nossa opção manter o item para os propósitos de pesquisa, uma vez que este factor ganha maior consistência teórica com a sua integração.

Seguiu-se o cálculo da validade discriminante para avaliar se os constructos são suficientemente distintos entre si (Marôco, 2014). Para o efeito, foram comparadas as VEM de cada factor com o quadrado da correlação entre os factores. Pela observação do modelo (Figura 1), verificamos que a correlação entre os factores Dor Emocional e Perda Relacional é $r$ DePr=.60. Assim, sendo a VEM $D e=.556$ e a VEM $P r=.573$ maiores do que $r^{2} \mathrm{DePr}=.36$, confirma-se que os dois factores têm validade discriminante. Por sua vez, a correlação entre os factores Dor Emocional e Evitamento é $r$ $D e E v i=.53$. Sendo a VEM De=.556, a VEM $E v i=.417$ e a $r^{2} D e E v i=.28$, é possível afirmar que o factor Dor Emocional apresenta validade discriminante uma vez que os valores de VEM são superiores aos de $r^{2}$. Por sua vez, o factor Evitamento também apresentou validade discriminante já que .417>.28. No que diz respeito à correlação entre os factores Perda Relacional e Evitamento, esta é $r$ PrEvi=.40. Assim, sendo a VEM $P r=.573$ e a VEM Evi=.417 maiores que $r^{2}$

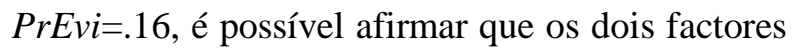
têm validade discriminante. Todos os valores obtidos evidenciam a satisfação plena dos critérios para aceitar a robustez da Escala de Sofrimento.

Foi também calculada a Fiabilidade Compósita (FC) deste modelo que estima a consistência interna dos itens reflexivos do constructo onde os valores devem ser iguais ou superiores a .7 para que a fiabilidade do mesmo seja apropriada, isto num intervalo entre 0 e 1 . Neste modelo multifactorial, o valor obtido de FC para o factor Dor Emocional foi de .789, para o factor Perda Relacional foi de .869 e para o factor Evitamento foi de .674. Com a excepção do Evitamento, todos os factores deste modelo apresentam FC apropriada. Optámos por manter o factor Evitamento, uma vez que há uma proximidade do valor de corte e, atendendo a que estes valores são recomendações e, por vezes, não há concordância entre os estudiosos quanto aos valores de critério. Com todos os factores contemplados a FC total do CGS foi de .923.

Foi ainda avaliado o índice de consistência interna através do Alfa de Cronbach. O factor Dor Emocional e o factor Evitamento apresentam uma consistência interna satisfatória (Alfa de Cronbach $=.781$ e .671 , respectivamente) e o factor Perda Relacional apresenta uma boa consistência interna (Alfa de Cronbach=.866), sendo o Alfa de Cronbach total $=.848$, o que revela boa consistência interna.

Perante isto, o modelo multifactorial ajustado com três factores (Dor Emocional, Perda Relacional e Evitamento), para a amostra do presente estudo, revelou uma qualidade de ajustamento bom para o $\chi^{2} / \mathrm{df}(1.609)$, para o CFI (.940) e para o GFI (.901) e um ajustamento aceitável para o RMSEA (.078). O valor de AIC é de 115.965 (Quadro 3). Estes dados mostram que este modelo apresentou um valor de AIC inferior aos modelos multifactorial original $(\mathrm{AIC}=139.074)$ e unifactorial (AIC=123.66).

Tendo optado pela estrutura de três factores, $\mathrm{o}$ cálculo dos mesmos foi feito do seguinte modo: o factor Dor Emocional é medido pelos itens 1 a 3 e diz respeito à experiência de sofrimento e outras emoções desagradáveis, vivenciadas no presente, relacionadas com a perda de alguém; o factor Perda Relacional é medido pelos itens 4 a 8 e refere-se às perdas relativas ao relacionamento e experiências partilhadas com a pessoa cuidada quando esta ainda era saudável; o factor Evitamento é medido pelos itens 9 a 11 e refere-se ao comportamento da pessoa com o objectivo de proteger-se da dor psicológica, tal como expressa nas emoções geradas nos contextos e relacionamentos psicossociais que, no caso dos enfermeiros, está directamente relacionada com o seu envolvimento emocional com os doentes.

\section{Discussão}

O objectivo deste estudo foi adaptar e validar a estrutura factorial da Escala de Sofrimento para Cuidadores (Caregiver Grief Scale), para os enfermeiros portugueses nos CSP. Tanto quanto sabemos, este é o primeiro estudo a testar a validade da CGS para enfermeiros na população portuguesa e na Europa. 
O modelo multifactorial original da CGS (Meichsner et al., 2016) avalia quatro factores do sofrimento: Dor Emocional, Perda Relacional, Perda Absoluta e Aceitação da Perda. A versão portuguesa desta escala (Vasconcelos-Raposo et al., 2019) validou um modelo unifactorial definido como sofrimento. No presente estudo, não foi possível confirmar a validade factorial da versão alemã com quatro factores nem da versão portuguesa com um factor para a população em estudo, mas foi possível fazê-lo com um modelo multifactorial de três factores (Dor Emocional, Perda Relacional e Evitamento).

O modelo original desenvolvido na Alemanha, inicialmente foi testado com três factores, apresentando valores de $\chi^{2} / \mathrm{df}=2.231$; $\mathrm{CFI}=.913$; RMSEA=.104 e $\mathrm{AIC}=3776.695$, tendo sido rejeitado por apresentar o valor de RMSEA superior a .10. Foi depois testado e validado para a mesma amostra com quatro factores apresentando índices de qualidade de ajustamento mais adequados: $\quad \chi^{2} / \mathrm{df}=1.394 ; \quad \mathrm{CFI}=.961$; RMSEA=.072 e AIC=3436.165 (Meichsner et al., 2016). No nosso estudo aconteceu precisamente o processo inverso, ou seja, em primeiro lugar foi testado um modelo com quatro factores que foi rejeitado em função dos índices de qualidade de ajustamento apresentados. Posteriormente, foi testado e validado com três factores, tendo-se obtido bons indicadores. Isto poderá ser explicado, talvez pelas diferenças culturais ou pelo facto de a nossa amostra ser apenas constituída por profissionais de saúde (cuidadores formais), estando assim reflectidos, concomitantemente, os aspectos da herança sociocultural que cada sujeito possui enquanto membro da mesma sociedade e submetidos a processos de enculturação e socialização semelhantes, assim como os valores inerentes à classe profissional a que pertencem. Estes aspectos diferem, qualitativamente, da amostra de cuidadores formais e informais estudados por Vasconcelos-Raposo et al. (2019). Importa ainda realçar que, quer os cuidadores informais como os formais que geraram a estrutura unifactorial, não tinham recebido treino específico para cuidar, ao contrário da nossa amostra.

No decorrer deste estudo, e após determinar as características psicométricas desta escala através de uma AFE, foram confirmados os resultados com uma AFC. Este procedimento permite uma análise mais adequada, além de permitir validar a estrutura factorial. A versão portuguesa para enfermeiros apresenta bons índices de consistência interna, com um Alfa de Cronbach total de .848. Para fins de comparação com estudos que possam recorrer à escala previamente validade por Vasconcelos-Raposo et al. (2019) que encontrou uma FC de .961, calculámos a FC considerando todos factores e cujos resultados evidenciaram um valor de .923 . A versão alemã também revela boas propriedades psicométricas, nomeadamente consistência interna total elevada (Alfa de Cronbach=.89) e alta confiabilidade de teste-reteste, visto que a correlação entre as pontuações totais da CGS seis meses após a primeira aplicação foi de .998 ( $p=.001)$. A versão portuguesa para cuidadores formais e informais apresentou também um índice de consistência interna elevado (Alfa de Cronbach=.936) e FC adequada (.934). Os Alfa de Cronbach foram próximos do modelo multifactorial original (.89) e do unifactorial (.936), tal como os valores de FC na amostra de enfermeiros (.923) e de cuidadores formais e informais (.934). O modelo em estudo apresentou índices de qualidade de ajustamento global ligeiramente mais elevados: $\chi^{2} / \mathrm{df}=1.609$; CFI $=.940 ; \quad$ GFI $=.901 ; \quad$ RMSEA $=.078 \quad \mathrm{e}$ $\mathrm{AIC}=115.965$. Estes resultados, possivelmente, podem ser explicados pelo facto dos autores que propuseram o modelo multifactorial original terem utilizado uma amostra de cuidadores de pessoas idosas com demência e no modelo unifactorial a amostra ser constituída por cuidadores de qualquer pessoa, independentemente da idade ou problema de saúde. Neste estudo, a amostra foi apenas constituída por profissionais de saúde, nomeadamente enfermeiros, que exercem a sua actividade profissional nos CSP. Para a amostra de enfermeiros, os dois modelos apresentaram-se aceitáveis, o unifactorial corrigido e o trifactorial. No entanto, o último foi o que apresentou indicadores mais robustos o que também se reflecte no AIC obtido: o mais baixo. Tendo em atenção o treino técnico e científico que os enfermeiros recebem, será espectável que o sofrimento seja percepcionado de forma multifactorial.

$\mathrm{O}$ instrumento em estudo apresenta validade factorial, uma vez que os pesos factoriais estandardizados de todos os itens são superiores a 
.5 , o que significa que os itens deste instrumento são o reflexo do factor latente (Sofrimento) que se pretendia medir. A validade convergente e a validade discriminante também se mostraram adequadas, uma vez que se verificou a existência de correlações positivas e elevadas entre os itens, sendo a VEM de todos os factores superior ao quadrado da correlação entre esses factores.

A amostra do presente estudo evidencia que as mulheres assumem um papel primordial no acto de cuidar, visto que no total da amostra em estudo existem apenas $10 \%$ de enfermeiros do sexo masculino, comparativamente com $90 \%$ do sexo feminino. $\mathrm{O}$ desequilíbrio da amostra pode ser justificado pelos papéis estabelecidos socialmente em relação ao homem e à mulher (Marins, Hansel, \& Silva, 2016), sendo uma constatação que a profissão de enfermagem é maioritariamente exercida por mulheres. $\mathrm{O}$ estudo da invariância por sexo pode ser um aspecto relevante a estudar futuramente, no sentido de perceber até que ponto o modelo proposto neste estudo se comporta de igual modo, tanto no sexo masculino como no sexo feminino. No entanto, para este efeito, os estudos a realizar deverão ter uma amostragem de âmbito nacional, uma vez que os desequilíbrios entre os profissionais por sexo são significativos.

Consideramos que os resultados do presente estudo devem ser analisados à luz de algumas limitações, nomeadamente ser representativo de uma só região do país e limitada aos profissionais de uma ACeS. Como sugestões futuras, seria pertinente replicar este estudo a outras regiões do país e aos enfermeiros que exercem funções nos cuidados diferenciados, para assim se tornar representativo da população portuguesa e ampliar a validade da CGS. Seria também pertinente continuar o estudo deste instrumento, uma vez que existem poucos instrumentos para avaliar o sofrimento de cuidadores formais, nomeadamente dos enfermeiros. A CGS apenas se encontra validada para cuidadores informais na população alemã e para cuidadores formais e informais portugueses, estando ainda em curso a validação para a população inglesa, tal como se pode constatar na revisão da literatura (Meichsner et al., 2016). Seria também interessante alargar este estudo a outras populações e outras culturas, pois como já foi referido, o grau de sofrimento varia de cultura para cultura, pelo que seria importante perceber as especificidades deste constructo, ou seja, quais as diferentes percepções de sofrimento entre os diversos países, bem como entre os cuidadores formais e informais.

Os resultados obtidos com o estudo desta escala podem ajudar a compreender melhor o contexto emocional dos cuidadores formais e a intervir junto deles. A compreensão deste fenómeno pode ainda fornecer aos gestores das instituições de saúde, um instrumento de gestão e avaliação do sofrimento dos seus colaboradores, para que possam ser tomadas medidas e implementadas estratégias proactivas que visem a melhoria da qualidade de vida e potenciem a motivação destes profissionais, com consequentes ganhos de produtividade, eficiência, melhor rentabilização de recursos humanos e melhor qualidade dos cuidados de enfermagem. Importa, ainda, ter presente que continua a ser necessário prosseguir com o processo de validação, nomeadamente nos aspectos que se prendem com a comparação com outros instrumentos que também avaliem o sofrimento. Porém, para os propósitos do presente estudo não foi identificado um instrumento que estivesse devidamente validado para a população em causa. Finalmente, urge, também, que se aprofundem as questões associadas com a validade de constructo tendo por base as condicionantes socioculturais.

\section{Conclusão}

No presente estudo, foi validada a estrutura factorial da Escala de Sofrimento para Cuidadores (Caregiver Grief Scale) com um modelo de três factores (Dor Emocional, Perda Relacional e Evitamento), o que contraria a versão original em alemão, assim como a validação prévia em português. Os valores encontrados são suficientemente robustos para a realização de estudos nomotéticos em enfermeiros nos Cuidados de Saúde Primários.

\section{Referências}

Adimando, A. (2018). Preventing and alleviating compassion fatigue through self-care: An educational workshop for nurses. Journal of 
Holistic Nursing, 36(4), 304-317. doi:10.1177/0898010117721581

Adwan, J. Z. (2014). Pediatric nurses' grief experience, burnout and job satisfaction. Journal of Pediatric Nursing, 29(4), 329-336. doi:10.1016/j.pedn.2014.01.011

Allie, Z., le Roux, E., Mahlatsi, K., Mofokeng, B., Ramoo, Z. A., Sibiya, K., . . Brits, H. (2018). Bereavement overload and its effects on, and related coping mechanisms of health care providers and ward administrators at National District Hospital in Bloemfontein, Free State. African Journal of Primary Health Care \& Family Medicine, 10(1). doi:165210.4102/phcfm.v10i1.1652

Almeida, F. D., de Moraes, M. S., \& Cunha, M. L. D. (2016). Taking care of the newborn dying and their families: Nurses' experiences of neonatal intensive care. Revista da Escola de Enfermagem da USP, 50, 118-124. doi:10.1590/S0080-623420160000300018

Borges, E. (2005). O sofrimento dos enfermeiros em Pediatria. Nascer e Crescer, 14(2), 123125.

Caldeira, L., \& Santos, M. (2019). Perfil do profissional de enfermagem na oncologia que vivencia o sofrimento moral. Revista Saúde, 13(2), ESP.49.

Campos, J., David, H., \& Souza, N. (2014). Prazer e sofrimento: Avaliação de enfermeiros intensivistas à luz da psicodinâmica do trabalho. Escola Anna Nery, 18(1), 90-95. doi:10.5935/1414-8145.20140013

Cerqueira, M. M. (2011). A pessoa em fim de vida e família: $O$ processo de cuidados face ao sofrimento (Tese de doutoramento). Universidade de Lisboa, Lisboa. Disponível em http://repositorio.ul.pt/handle/10451/3614

Chan, D., Livingston, G., Jones, L., \& Sampson, E. L. (2012). Grief reactions in dementia carers: A systematic review. International Journal of Geriatric Psychiatry, 28(1), 1-17. doi:10.1002/gps.3795

Coelho, M. E., \& Ferreira, A. C. (2015). Cuidados paliativos: Narrativas do sofrimento na escuta do outro. Revista Bioética, 2, 340. doi:10.1590/1983-80422015232073

Conselho Internacional de Enfermeiros (2008). Servir a comunidade e garantir qualidade: $O s$ enfermeiros na vanguarda dos Cuidados de Saúde Primários. Genebra: ICN.

Encarnação, P., Oliveira, C. C., \& Martins, T. (2015). Dor e sofrimento conceitos entrelaçados: Perspetivas e desafios para os enfermeiros. Cuidados Paliativos, 2(2), 22-31. Disponível em http://www.apcp.com.pt/uploads/Revista_Cui dados_Paliativos_v2_n2.pdf

Gama, M. (2013). O luto profissional nos enfermeiros (Tese de doutoramento). Instituto de Ciências da Saúde da Universidade Católica Portuguesa, Lisboa. Disponível em http://repositorio.ucp.pt/handle/10400.14/13973

Hedler, H. C., Santos, M. J., Faleiros, V. P., \& Almeida, M. A. (2016). Representação social do cuidado e do cuidador familiar do idoso. Revista Katálysis, 19(1), 143-153. Disponível em http://www.scielo.br/pdf/rk/v19n1/14144980-rk-19-01-00143.pdf

Krikorian, A., Limonero, J. T., Román, J. P., Vargas, J. J., \& Palacio, C. (2014). Predictors of suffering in advanced cancer. The American Journal of Hospice \& Palliative Care, 31(5), 534-542. doi:10.1177/1049909113494092

Lin, C. H., Liu, Y. C., \& Chiang, H. H. (2020). From self-compassion to compassionate action: Reflecting on ending life of stillbirth care in nursing. Scandinavian Journal of Caring Sciences. doi:10.1111/scs.12842

Lindberg, D. (2012). When your patients die: Living with cumulative grief. ONS Connect, 27(10), 10-14. Disponível em https://europepmc.org/article/med/23094446

Luzardo, A. R., \& Waldman, B. F. (2004). Atenção ao familiar cuidador do idoso com doença de Alzheimer. Acta Scientiarum, 26(1), 135-145. Disponível em http://www.lume.ufrgs.br/bitstream/handle/10 183/140057/000590924.pdf?sequence $=1$

Machado, A. G., \& Melro A. R. (2008). Cuidadores: Seus amores e suas dores. Psicologia \& Sociedade, 20(3), 444-452. doi:10.1590/S0102-71822008000300015

Marins, A. M., Hansel, C. G., \& Silva, J. (2016). Mudanças de comportamento em idosos com doença de Alzheimer e sobrecarga para o cuidador. Escola Anna Nery, 20 (2), 352-356. doi:10.5935/1414-8145.20160048 
Marôco, J. (2014). Análise das equações estruturais: Fundamentos teóricos, software \& aplicações. Cruz Quebrada: ReportNumber.

Meichsner, F., Schinköthe, D., \& Wilz, G. (2016). The caregiver grief scale: Development, exploratory and confirmatory factor analysis, and validation. Clinical Gerontologist, 39(4), 342-361. doi:10.1080/07317115.2015.1121947

Morck, A. C., \& Moules, N. J. (2019). Do you think the angels will speak spanish? Nurses' experiences of death in pediatric oncology. Journal of Applied Hermeneutics. Retrieved from < Go to ISI $>$ ://WOS:000493193800012

Oliveira, F. C., Cleveland, L. M., Darilek, U., Silva, A. R. B., \& Carmona, E. V. (2018). Brazilian neonatal nurses' palliative care experiences. Journal of Perinatal \& Neonatal Nursing, 32(4), E3-E10. doi:10.1097/Jpn.0000000000000361

Paula, G., Fontes-Reis, J., Conceição-Dias, L., Damásio-Dutra, V., Souza-Braga, A., \& Antunes-Cortez, E. (2010). O sofrimento psíquico do profissional de enfermagem da unidade hospitalar. Aquichan, 10(3), 267279. doi:10.5294/aqui.2010.10.3.7

Peixoto, M. J., \& Borges, E. (2011). O sofrimento no contexto da doença. Revista Portuguesa de Enfermagem de Saúde Mental, 6, 36-39. Disponível em http://www.scielo.mec. pt/pdf/rpesm/n6/n6a06.pdf

Pimenta, Cl., Bezerra, T., Martins, K., Costa, T., Viana, L., Costa, M., \& Costa, K. (2020). Prazer e sofrimento entre enfermeiros do contexto hospitalar. Revista Brasileira de Enfermagem, 73(2), e20180820. doi:10.1590/0034-7167-2018-0820

Pires, L., Monteiro, M., \& Vasconcelos-Raposo, J. (2020). Sofrimento nos enfermeiros em cuidados de saúde primários. Revista de Enfermagem Referência, V(1), 1-10. doi:10.12707/RIV19096

Prieto, G., Muniz, J., Almeida, L., \& Bartram, D. (1999). Uso de los tests psicológicos em España, Portugal e Iberoamérica. Revista Iberoamericana de Diagnóstico y Evaluación - e Avaliação Psicológica, 8(2), 67-82. Disponível em https://www.researchgate.net/publication/259 692538_Uso_de_los_tests_psicologicos_en_E spana_Portugal_e_Iberoamerica
Reichenhim, M., \& Moraes, C. (2007). Operacionalização de adaptação transcultural de instrumentos de aferição usados em epidemiologia. Revista de Saúde Pública, 41(4), 665-673. Disponível em https://www.scielo.br/pdf/rsp/v41n4/6294.pdf

Rodrigues, J. O., \& Silva, E. A. (2016). O prazer e o sofrimento dos homens no trabalho em serviços de saúde. Revista de Enfermagem UFPE on line, 10(7), 2544-2554. doi:10.5205/reuol.9106-80230-1SM1007201631

Schaefer, R. (2017). Sofrimento moral: Avaliação de risco em enfermeiros (Tese de doutoramento). Instituto de Ciências da Saúde da Universidade Católica Portuguesa, Porto. Disponível em http://hdl.handle.net/10400.14/24202

Seabra-Santos, M., Almiro, P., Simões, M., \& Almeida, L. (2019). Testes psicológicos em Portugal: Atitudes, problemas e perfil de utilizadores. Revista Iberoamericana de Diagnóstico y Evaluación - e Avaliação Psicológica, 53(4), 101-112. doi:10.21865/RIPED53.4.08

Silva, N. J. R., \& Neto, H. V. (2018). Levels of stress, professional exhaustion and coping in nursing professionals from a palliative care service in acute situations. International Journal on Working Conditions, 15, 96-112. Retrieved from <Go to ISI $>$ ://WOS:000441336400007

Travelbee, J. (2004). Modelo de relação pessoa-apessoa. In A. Marriner Tomey, Teóricas de enfermagem e a sua obra: Modelos e teorias de enfermagem ( $5^{\mathrm{a}}$ ed., pp. 467-479). Loures: Lusociência.

Vasconcelos-Raposo, J., Ribeiro, A. M., Silva, A. R., Santos, B., \& Teixeira, C. M. (2019). Validity of the portuguese version of the Caregiver Grief Scale. PsychTech \& Health Journal, 3(1), 30-42. doi:10.26580/PTHJ.art19-2019.

Vega, V. P., Gonzalez, R. R., Bustos, M. J., Rojo, S. L., Lopez, E. M. E., Rosas, P. A., \& Hasbun, I. C. G. (2017). Relationship between grief support and burnout syndrome in professionals and technicians of pediatric health. Revista Chilena De Pediatria-Chile, 88(5), 614-621. 
doi:10.4067/S0370-41062017000500007

Viana, A. (2014). Sofrimento experienciado pelo enfermeiro quando cuida o doente em fim de vida com dor não controlada (Dissertação de mestrado). Escola Superior de Saúde do Instituto Politécnico de Viana do Castelo, Viana do Castelo. Disponível em http://repositorio.ipvc.pt/bitstream/20.500.119 60/1237/1/Aurora_Viana.pdf

Wenzel, J., Shaha, M., Klimmek, R., \& Krumm, S. (2011). Working through grief and loss: Oncology nurses' perspectives on professional bereavement. Oncology Nursing Forum, 38(4), E272-282.

doi:10.1188/11.ONF.E272-E282

Wisekal, A. E. (2015). A concept analysis of nurses' grief. Clinical Journal of Oncology Nursing, 19(5), E103-107.

doi:10.1188/15.CJON.E103-E107 